\title{
Clinical and biochemical characteristics of Papillary Thyroid Cancer (PTC) according to the presence of BRAF (V600E) mutation
}

Amelia Oleaga ${ }^{1}$, Fernando Goñi ${ }^{1}$, Miguel Paja ${ }^{1}$, Maite Perez de Ciriza ${ }^{1}$, Eider Etxeberría ${ }^{1}$, Laura Calles ${ }^{1}$, Estíbaliz Ugarte ${ }^{1}$, Aitziber Ugalde ${ }^{2}$, Ramón Elorza ${ }^{1}$. Endocrinology Department ${ }^{1}$, Pathology Department ${ }^{2}$. Universitary Basurto Hospital. Bilbao. Spain.

\section{Introduction:}

BRAF (V600E) mutation is the most frequent detected genetic change in PTC. Its presence has been related to aggressive clinical and pathological features. Therefore, BRAF mutation has drawn considerable interest as a potential prognostic factor for PTC. The presence of the mutation confers the tumour a disability to uptake RAI, diminishing the therapeutic tools. However if this feature is related to some kind of tumour dedifferentiation is not known. The aim of this study was to explore if there were any differences in preoperative thyroglobulin (PTg) levels and the presence of autoimmunity (PTg Ab), among patients harbouring the mutation and those who didn't.

\section{Description of methods/Design:}

We evaluated 62 patients (51 females) with pathological diagnosis of PTC. All of them underwent total thyroidectomy, 55 central lymph node dissection (CLND), and 13 lateral neck dissection as well. DNA was extracted from neoplastic cells and BRAF mutation was detected by PCR and sequencing. Analysis included age, preoperatively TSH, PTg and PTg Ab, tumour size and thyroid weight.

\begin{tabular}{|l|c|c|c|}
\hline & BRAF + & BRAF & $P$ \\
\hline $\mathrm{N}$ & $32(51,6 \%)$ & $30(48,4 \%)$ & \\
\hline Sex (M/F) (51/11) & $5 / 27$ & $6 / 24$ & 0,68 \\
\hline Mean Age (years) & 48,9 & 50,4 & 0,71 \\
\hline Tumour size (mm) & 16,8 & 19,4 & 0.43 \\
\hline Thyroid weight (g) & 19,5 & 23 & 0,31 \\
\hline TSH(mU/L) & 3,26 & 2,33 & 0,14 \\
\hline PTg Ab positive(\%) & 33,3 & 29,6 & 0,6 \\
\hline PTg (ng/mL) & 154,5 & 355,6 & 0,13 \\
\hline
\end{tabular}

\section{Conclusions}

In our series, harbouring BRAF mutation, neither implies less production of $\mathrm{Tg}$ by the tumour, nor higher frequency of autoimmunity, although there is a tendency towards less PTg concentration and smaller tumour size. Nevertheless higher PTg levels may be related to a trend to heavier thyroid gland. In view of these results, and considering the small size of the sample, we can't conclude that the presence of BRAF mutation, involves any grade of dedifferentiation in PTC. 